\title{
Electronic spectroscopy, stimulated emission, and persistent spectral hole burning of cryogenic nitrogen matrices doped with tetrabenzoporphin
}

\author{
S. M. Arabei \\ Belarusian State Agrarian and Technical University, Minsk, Belarus \\ J.-P. Galaup ${ }^{\text {a) }}$
}

Laboratoire Aimé Cotton, CNRS \& Université Paris-Sud 11, Orsay, France

J. G. McCaffrey

Department of Chemistry, National University of Ireland, Maynooth, Ireland

\section{N. Shafizadeh and C. Crépin}

Institut des Sciences Moléculaires d'Orsay, CNRS \& Université Paris-Sud 11, Orsay, France (Submitted April 20, 2012)

Fiz. Nizk. Temp. 38, 917-922 (August 2012)

\begin{abstract}
This paper deals with our on-going work on the electronic spectroscopy of tetrapyrrole molecules embedded in cryogenic hosts. Under nanosecond laser excitation of free-base tetrabenzoporphin molecules in a nitrogen matrix at $8 \mathrm{~K}$, increasing laser pulse energy results in essential enhancement of the intensity of one or several vibronic emission lines in the fine-structure fluorescence spectrum. Some characteristics of stimulated radiation are realized in the observed intense monochromatic radiation as a result of a transition from the purely electronic $S_{1}$ level to a vibrational sublevel of the $S_{0}$ state, corresponding to the excitation of C-C stretching vibrations of tetrapyrrole methane bridges. We also report on persistent spectral hole-burning effects. Hole and antihole formation analysis gives some insight on the photochemical properties of tetrabenzoporphin in a nitrogen matrix. (C) 2012 American Institute of Physics. [http://dx.doi.org/10.1063/1.4746794]
\end{abstract}

\section{Introduction}

Development of solid-state laser-active media based on dyes continues to be one of the important problems of modern high technology. It is stimulated, first of all, by the need to create micron-size lasers. The problem of miniaturization of solid-state elements motivates the study of spectral and kinetic characteristics of spontaneous and induced (stimulated) radiation of thin-film colored materials. Studies of thin $1 \mu \mathrm{m}$ silica gel slabs doped with dyes of different classes (rhodamines, oxazole derivatives, coumarins, porphyrins, etc.) showing lasing characteristics ${ }^{1-6}$ are described in the literature. It is important to note that in some experiments ${ }^{3,5}$ external optical resonator mirrors were missing, but stimulated emission (SE) was nevertheless detected due to light amplification in a single pass through the layer by just a "traveling wave" (traveling lasing wave).

We have performed spectroscopic studies of free base phthalocyanine $\left(\mathrm{H}_{2} \mathrm{Pc}\right)$ and its zinc counterpart $(\mathrm{ZnPc}){ }^{7}$ as well as of free base tetrabenzoporphin $\left(\mathrm{H}_{2} \mathrm{TBP}\right)^{8}$ in matrices of rare gases $(\mathrm{Ne}, \mathrm{Ar}, \mathrm{Kr}, \mathrm{Xe})$ at low temperature $(8 \mathrm{~K})$. We found that under the action of pulsed-laser excitation, SE was observed in guest molecules in these matrices and corresponds to transitions between the lowest singlet state $S_{1}$ and specific vibrational levels of the ground electronic state $S_{0}$ $\left(S_{1}(0-0) \rightarrow S_{0}(0-1)\right.$ transition). The interest in porphyrin and phthalocyanine molecules as systems capable of SE is due to their high fluorescence quantum yields, high thermal and photo stabilities, and intense absorption bands in the visible spectrum, which allow them to effectively carry out their excitation and localization of the fluorescence spectra in the red and near-IR region. We recall that lasing emission from an organic molecule was observed for the first time in 1964 by Sorokin and Lankard: ${ }^{9,10}$ stimulated emission was observed when a solution of chloro-aluminum phthalocyanine dissolved in ethanol was irradiated by a beam from a giant-pulsed ruby laser. The wavelength of this stimulated emission was centered at approximately $755 \mathrm{~nm}$.

The present work is a logical continuation of previously published results, ${ }^{7,8}$ in which the authors discussed the occurrence of the phenomenon of $\mathrm{SE}$ in $\mathrm{H}_{2} \mathrm{Pc}, \mathrm{ZnPc}$, and $\mathrm{H}_{2}$ TBP molecules isolated in cryogenic matrices of rare gases, identifying a number of problems, so far-unsolved.

For instance, a necessity arises to clarify the role of different parameters on the stimulated emission phenomenon by changing the nature of the host matrix, then changing the electron-phonon interaction. It is still unclear why the intensification of light emission only occurs in a very limited number of vibronic transitions. Therefore, it appears necessary to establish the critical values for achieving population inversion of levels, taking into account the "distribution" of fluorescence quantum yield between the vibronic transitions (i.e., the intensity of vibronic lines), the value of the relaxation rate, and the contribution of different normal modes in the vibrational levels of the ground state $S_{0}$.

The purpose of the present paper is to communicate new information on the spectral characteristics of $\mathrm{H}_{2}$ TBP molecules embedded in a low-temperature matrix of molecular nitrogen $\left(\mathrm{N}_{2}\right)$. The choice of $\mathrm{H}_{2} \mathrm{TBP}$, the structural formula for which is shown in Fig. 1, is for two main reasons. First, the quasi-line fluorescence spectra of $\mathrm{H}_{2} \mathrm{TBP}$ in $n$-octane 


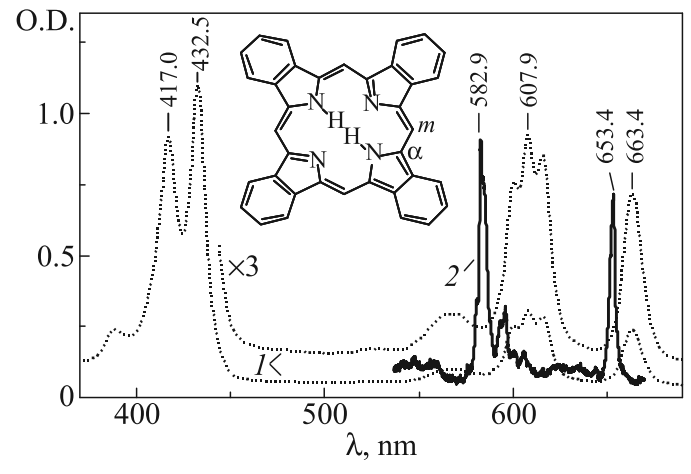

FIG. 1. Absorption spectra of $\mathrm{H}_{2} \mathrm{TBP}$ in pyridine at $293 \mathrm{~K}$ (1) and in a nitrogen matrix at $8 \mathrm{~K}(2)$. The inset shows the structure of $\mathrm{H}_{2} \mathrm{TBP}$.

Shpol'skii matrix at $4.2 \mathrm{~K}$ reveal lines assigned to individual vibronic transitions with exceptionally narrow spectral widths. For example, the components of the 0-0-doublet, recorded in the fluorescence spectrum of $\mathrm{H}_{2} \mathrm{TBP}$ in a single-crystal matrix of $n$-octane at $4.2 \mathrm{~K}$, have half-widths of 1.5 and $1.8 \mathrm{~cm}^{-1}$, respectively. ${ }^{11}$ Second, the role and the high speed of photochemical NH-tautomerism in $\mathrm{H}_{2} \mathrm{TBP},{ }^{12}$ which is the cause of efficient photoinduced interconversion of impurity centers in Shpol'skii matrices, ${ }^{13}$ needs to be studied further.

\section{Experimental}

In order to obtain low-temperature matrices of nitrogen doped with $\mathrm{H}_{2} \mathrm{TBP}$, a special device was designed and built to be attached to the cryostat. All the details have been fully described previously. ${ }^{8}$ The temperature of the heater, which could reach up to $300^{\circ} \mathrm{C}$, was adjusted for the $\mathrm{H}_{2}$ TBP powder. Sublimed porphyrin molecules were carried by the flow of nitrogen, forming a gaseous mixture of $\mathrm{N}_{2}+\mathrm{H}_{2} \mathrm{TBP}$, which was deposited on a sapphire window in a closed-cycle helium displex cryostat at $\sim 20 \mathrm{~K}$ to form a thin polycrystal film with a typical thickness of about $250 \mu \mathrm{m}$. Gas flow rates of $10 \mathrm{mmol} / \mathrm{h}$ allowed good matrix isolation of tetrapyrrole molecules as monomers, as monitored by the absorption spectrum of the prepared sample, which also allowed to estimate the resultant concentration of the dye. Fluorescence, stimulated emission, and fluorescence excitation spectra were produced with a pulsed dye laser at $10 \mathrm{~Hz}$ using DCM, Rh610, or Rh6G pumped by a frequency-doubled $\mathrm{Nd}^{3+}$ : YAG laser or an excimer $\mathrm{XeCl}$ laser. Fluorescence was recorded through an HRS 600 monochromator from HoribaJobin-Yvon using a high-speed iCCD-camera (ANDOR, model DH-720). Most of the measurements were made at about $8 \mathrm{~K}$.

\section{Results and discussion}

\section{Absorption}

The absorption spectrum of $\mathrm{H}_{2}$ TBP in pyridine at room temperature is shown by the dotted trace in Fig. 1 (curve 1).

According to Refs. 11, 14-16, the band at $663.4 \mathrm{~nm}$ belongs to the $S_{1} \leftarrow S_{0}$ transition ( $Q_{x}$ band), while the bands at 432.5 and $417.0 \mathrm{~nm}$ are assigned to the $S_{3} \leftarrow S_{0}$ and $S_{4}$ $\leftarrow S_{0}$ transitions ( $B_{x}$ and $B_{y}$ bands), respectively. The 580$620 \mathrm{~nm}$ region is where the $Q_{y}$ band is located, and whose absorption spectrum shows the presence of three peaks. Careful analysis of the data, taking into account polarization of the quasi-line spectra, showed that the contour of this band results from the contributions of two different types of transitions: the high-frequency vibronic transitions of the lowest $S_{1}$ electronic state, and the pure electronic $S_{2} \leftarrow S_{0}$ transition. Contemplation of the nature of the violation of mirror symmetry in frequencies, as well as in the activity of individual normal modes in the $S_{1}$ and $S_{0}$ states of $\mathrm{H}_{2} \mathrm{TBP}$, led to the conclusion that the purely electronic band assigned to the $S_{2} \leftarrow S_{0}$ transition corresponds to the central maximum of the complex recorded contour. ${ }^{14,15}$ Accordingly, an estimate of the energy difference $\Delta E_{S_{2} S_{1}}=E_{S_{2}}-E_{S_{1}}$ for $\mathrm{H}_{2}$ TBP in pyridine gives the value of $\sim 1370 \mathrm{~cm}^{-1} \cdot 11,14,16$ The same value, as well as similar band shape, were also observed for $\mathrm{H}_{2}$ TBP in DMSO. A noticeable change in the shape of the absorption band in the 580-620 nm region is observed in solvents such as $n$-octane $\left(\Delta E_{S_{2} S_{1}} \approx 1445\right.$ $\left.\mathrm{cm}^{-1}\right),{ }^{16}$ ethanol $\left(\Delta E_{S_{2} S_{1}} \approx 1480 \mathrm{~cm}^{-1}\right),{ }^{17}$ and in a mixture of tetrahydrofuran and ether, ${ }^{18}$ where the most intense feature is in the $580-620 \mathrm{~nm}$ range- the short-wavelength component of the complex structure.

New information is obtained on the spectral characteristics of $\mathrm{H}_{2}$ TBP molecules, whose inhomogeneous broadening is partially removed in their low-temperature absorption spectra in a solid $\mathrm{N}_{2}$ matrix (Fig. 1, curve 2). As can be seen, significant differences exist in the absorption spectrum of $\mathrm{H}_{2} \mathrm{TBP} / \mathrm{N}_{2}$ and the spectrum in pyridine. Compared with a pyridine matrix, the $\sim 10 \mathrm{~nm}$ blue-shift of absorption bands at $653.4 \mathrm{~nm}\left(=\lambda_{00}\right.$ of $\left.S_{1} \leftarrow S_{0}\right)$ is indicative of a weakening in the interaction between the molecules and the surrounding $\mathrm{N}_{2}$ matrix. Also, the half-width of the spectral bands is up to 3.5 times smaller. For example, the band of the purely electronic $S_{1} \leftarrow S_{0}$ transition becomes narrower, changing from $\sim 265 \mathrm{~cm}^{-1}$ in pyridine down to $\sim 75 \mathrm{~cm}^{-1}$ in $\mathrm{N}_{2}$. However, an accurate localization of the $S_{2} \leftarrow S_{0}$ transition of $\mathrm{H}_{2} \mathrm{TBP}$ in $\mathrm{N}_{2}$ is not well defined, in spite of a significant intensity of the absorption bands in the $570-600 \mathrm{~nm}$ region. Although this cannot be determined with great accuracy, the similarity between the absorption spectra of $\mathrm{H}_{2} \mathrm{TBP}$ in $\mathrm{N}_{2}$ and in $n$ octane matrix is noticeable, as both these spectra have an intense band in the short wavelength range $580-620 \mathrm{~nm}$. The spectral separation between this intense band and the band assigned to the $S_{1} \leftarrow S_{0}$ electronic transition is equal to $\sim 1750 \mathrm{~cm}^{-1}$ in the $n$-octane matrix and $\sim 1850 \mathrm{~cm}^{-1}$ in the $\mathrm{N}_{2}$ matrix. This observation allows us to infer that the exact $\Delta E_{S_{2} S_{1}}$ for $\mathrm{H}_{2} \mathrm{TBP} / \mathrm{N}_{2}$ should be slightly greater than that in n-octane Shpol'skii matrix, for which $\Delta E_{S_{2} S_{1}} \approx 1445 \mathrm{~cm}^{-1}$. A recent investigation of phthalocyanine in inert gas matrices ${ }^{19}$ has similarly concluded that $\Delta E_{S_{2} S_{1}}$ was slightly larger for $\mathrm{H}_{2} \mathrm{Pc} / \mathrm{N}_{2}$ than for $\mathrm{H}_{2} \mathrm{Pc}$ trapped in the $n$-decane Shpol'skii matrix.

\section{Stimulated emission and its excitation}

Upon excitation in the $Q_{y}$ absorption band at $\lambda_{\text {exc }}$ $=582.7 \mathrm{~nm}$ with the pulsed dye laser $\left(P_{\text {exc }} \approx 10 \mathrm{~mW}\right.$, pulse length $=10 \mathrm{~ns})$, the fluorescence spectrum of $\mathrm{H}_{2} \mathrm{TBP}$ in $\mathrm{N}_{2}$ at $8 \mathrm{~K}$ has the form shown in Fig. 2 (curve 1). An intense band assigned to the pure electronic $S_{1} \rightarrow S_{0}$ transition occurs at $653.8 \mathrm{~nm}$, followed by low-intensity bands corresponding to vibronic transitions covering the $660-740 \mathrm{~nm}$ region. The resulting spectrum resembles the quasi-line 


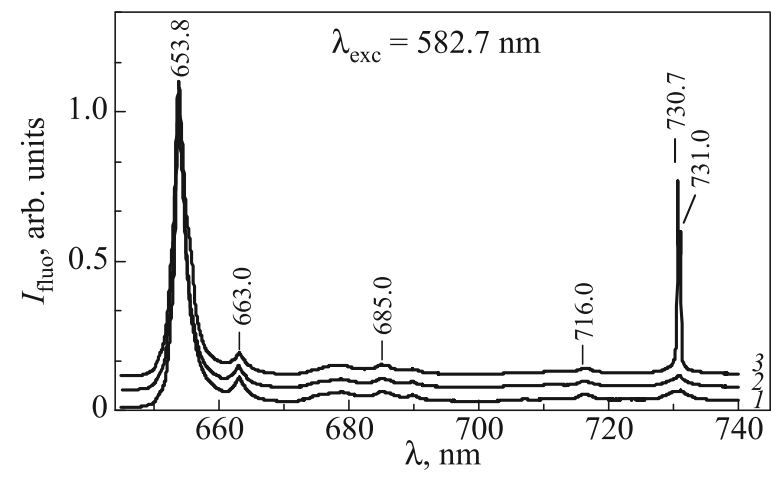

FIG. 2. Fluorescence and stimulated emission spectra of $\mathrm{H}_{2} \mathrm{TBP}$ molecules in a nitrogen matrix at $8 \mathrm{~K}\left(\lambda_{\mathrm{exc}}=582.7 \mathrm{~nm}, P_{\text {laser }} \approx 10(1), 30(2)\right.$ and 100 (3) $\mathrm{mW}$.

fluorescence spectrum of $\mathrm{H}_{2} \mathrm{TBP}$ in $n$-octane at $4.2 \mathrm{~K},{ }^{16}$ where the most intense vibronic band is a band corresponding to a low-frequency normal mode at $\sim 220 \mathrm{~cm}^{-1}$ (Fig. 2, see the band at $663.0 \mathrm{~nm}$ ). As the power of pulsed laser excitation is increased from $P_{\text {laser }} \approx 30 \mathrm{~mW}$ to $100 \mathrm{~mW}$, two intense lines $(730.7$ and $731.0 \mathrm{~nm})$ from the weak vibronic band at $731 \mathrm{~nm}$ of the normal fluorescence spectrum increase drastically (Fig. 2, compare curves 2 and 3). Attention is drawn to the sharp narrowing of these lines (down to $\sim 5 \mathrm{~cm}^{-1}$, which is the resolution limit of our spectrometer) and to their dependence on the relative intensities of laser excitation power. These lines are assigned to SE. Changing the laser excitation wavelength in the region $580-585 \mathrm{~nm}$ does not lead to the appearance of other new SE lines in the recorded spectrum. There is only a slight difference in the relative intensities of the lines at 730.7 and $731.0 \mathrm{~nm}$. Similarly to the observations reported in Refs. 7 and 8, we can conclude that emission at these wavelengths is strongly enhanced when the exciting laser intensity exceeds a certain threshold value. This means that an inversion of population is achieved between the lowest electronic state $S_{1}$ and some vibrational sublevels of the ground state $S_{0}$. When such a situation arises, stimulated emission can occur and overpass any spontaneous fluorescence. In the case of $\mathrm{H}_{2} \mathrm{TBP} / \mathrm{N}_{2}$, the analysis of the spectral data shows that SE takes place on a vibronic transition that involves a vibrational sublevel with energy of about $1615 \mathrm{~cm}^{-1}$ from the ground $S_{0}$ state. This vibrational sublevel corresponds to the excitation of valence vibrations involving the $\mathrm{C}_{\alpha} \mathrm{C}_{m}$ methine bridges (see Fig. 1) with $B_{1 g}$ symmetry. ${ }^{11,20}$ It is important to note that $\mathrm{SE}$ is observed under pulsed laser excitation, with whose duration it does not exceed the lifetime of the $S_{1}$ state (for example, in the case of $\mathrm{H}_{2}$ TBP in an ethanol-ether mixture, $\tau_{S}=11 \mathrm{~ns}$ (Ref. 21)), and at a repetition rate of $10 \mathrm{~Hz}$, i.e., $100 \mathrm{~ms}$ between successive pulses. Such a situation precludes an effective population of the triplet state, well known for its role as "traps" for the active sites, which should be the case under steady-state photoexcitation. It is then difficult to achieve conditions for population inversion.

In order to clarify the conditions under which SE occurs in $\mathrm{H}_{2} \mathrm{TBP} / \mathrm{N}_{2}$ we studied the excitation spectra of stimulated emission. Figure 3(a) shows such spectra for the $Q_{y}$ band region at $8 \mathrm{~K}$ (curve 1 , monitoring emission at $\lambda_{\mathrm{obs}}=730.7 \mathrm{~nm}$, and curve 2 for $\lambda_{\text {obs }}=731.0 \mathrm{~nm}$ ), which is compared with the absorption spectrum of $\mathrm{H}_{2} \mathrm{TBP} / \mathrm{N}_{2}$ (curve 3) in the same
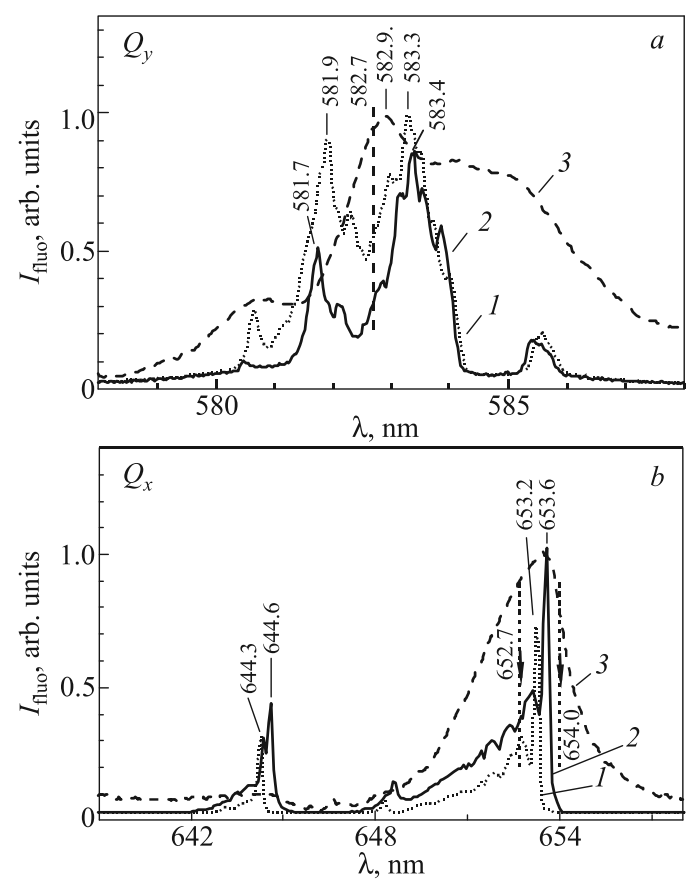

FIG. 3. Comparison of the excitation spectra of stimulated emission ( 1 and 2) and the absorption spectrum (3) of $\mathrm{H}_{2} \mathrm{TBP}$ molecules in a nitrogen matrix at $8 \mathrm{~K}$ for $S_{2} \leftarrow S_{0}\left(\lambda_{\text {obs }}=730.7\right.$ (1) and 731.0 (2) $\mathrm{nm}$ ) (a) and $S_{1} \leftarrow S_{0}$ $\left(\lambda_{\text {obs }}=730.7\right.$ (1) and $\left.731.1(2) \mathrm{nm}\right)(\mathrm{b})$.

region. With the detection of emission in each of the two observed SE components it can be seen that the contours of the bands in the excitation spectra of SE are almost identical, and their spectral shift relative to each other is negligible. However, the maxima of the bands in the excitation spectra of SE do not coincide with the maximum absorption spectrum in this area, observed at $582.9 \mathrm{~nm}$. This can be considered as a consequence of the population inversion, induced at the wavelengths 580 $585 \mathrm{~nm}$ and excitation powers of up to $100 \mathrm{~mW}$, which was used for the two types of impurity centers. It should be noted that SE intensity at $730.7 \mathrm{~nm}$ is higher than SE intensity at $731.0 \mathrm{~nm}$. This is true for almost all excitation wavelengths. An explanation of the observed features of the excitation spectra of SE for each type of impurity centers requires a detailed analysis of the electronic and electronic-vibrational levels of the $Q_{x}$ and $Q_{y}$ states involved in the active transitions. ${ }^{11,16}$

Stimulated emission around $731 \mathrm{~nm}$ is also observed under pulsed laser excitation in the long wavelength absorption $Q_{x}$ band of $\mathrm{H}_{2} \mathrm{TBP} / \mathrm{N}_{2}$ (Fig. 4). However, unlike in the

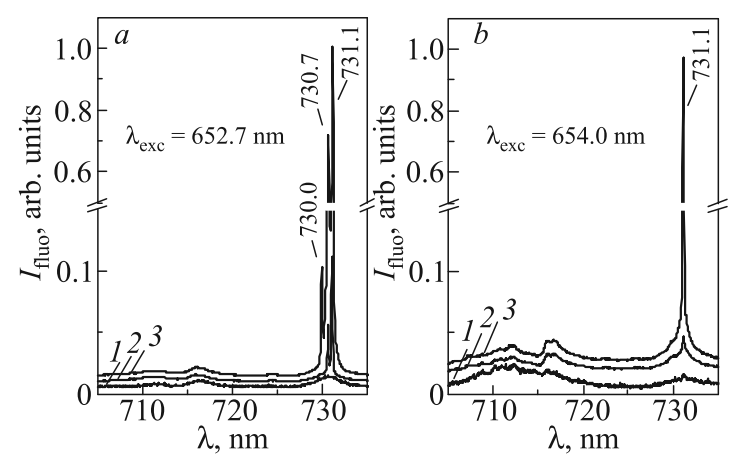

FIG. 4. Fluorescence and stimulated emission spectra of $\mathrm{H}_{2} \mathrm{TBP}$ molecules in a nitrogen matrix at $8 \mathrm{~K}\left(\lambda_{\mathrm{exc}}=652.7\right.$ (a) and 654.0 (b) $\mathrm{nm}, P_{\text {laser }} \approx 10$ (1), $30(2)$ and $100(3) \mathrm{mW})$. 
case of excitation in the $Q_{y}$ band, in this situation there is a dependence of the number of SE components on the change in $\lambda_{\text {exc }}$ within the $Q_{x}$ absorption band (Fig. 3(b), the spectral position of the laser exciting radiation is shown by the arrows and vertical dotted lines).

At $\lambda_{\text {exc }}=652.7 \mathrm{~nm}$ and under laser excitation power gradually increasing up to $P_{\text {laser }} \approx 100 \mathrm{~mW}$, four SE components appear in the emission spectrum (Fig. 4(a)), while at $\lambda_{\text {exc }}=654.0 \mathrm{~nm}$ only one SE component is intensified (Fig. 4(b)). An explanation of this behavior observed in the spectral composition of the SE is provided by an analysis of the excitation spectra of the SE in the $Q_{x}$ band absorption (Fig. 3(b)). As shown in Fig. 3(b), the broad $Q_{x}$ absorption band of $\mathrm{H}_{2} \mathrm{TBP} / \mathrm{N}_{2}$ at $8 \mathrm{~K}$ (curve 3) is transformed into a structured band in the SE excitation spectrum.

For example, it can be noticed that for $\lambda_{\mathrm{obs}}=731.1 \mathrm{~nm}$, the SE emission line component is on the long-wavelength side of the excitation spectrum of the SE (curve 2); there is a narrow intense line at $653.6 \mathrm{~nm}$ and a broad and structured band on its short-wavelength side. Clearly, such a structure in the SE excitation spectrum is formed by the excitation (absorption) of the narrow zero-phonon line (ZPL) corresponding to the $S_{1} \leftarrow S_{0}(0,0)$ transition and to its broad phonon side band (PSB). A similar observation can be made regarding the shorter-wavelength component of the SE spectrum recorded at $\lambda_{\text {obs }}=730.7 \mathrm{~nm}$ (curve 1 ), while a slight blue shift can also be noticed. The observed SE excitation spectra may explain the dependence on $\lambda_{\text {exc }}$ of the structured components. The excitation on the short-wavelength side within the $Q_{x}$ band induces a simultaneous photoactivation of "red" (via their PSB) and "blue" (through their ZPL) kinds of impurity centers, whereas the excitation on the long-wavelength side affects only the "red" impurity centers through their ZPL. Indeed, in the first case, four types of impurity centers are identified, exhibiting SE at 731.1, 730.7, 730.3 and $730.0 \mathrm{~nm}$, respectively (Fig. 4(a)), while in the second case, only one SE component at $731.1 \mathrm{~nm}$ can be induced (Fig. 4(b)). Notice that in the resulting SE excitation spectra shown in Fig. 3(b), SE is observed upon excitation in the $Q_{x}(0-0)$ band of the purely electronic transition, and also in the vibronic $Q_{x}(0-1)$ band at $\sim 644 \mathrm{~nm}$.

The obtained results demonstrate that under relatively high-power pulsed laser excitation in the $Q_{x}$ and $Q_{y}$ band of $\mathrm{H}_{2} \mathrm{TBP} / \mathrm{N}_{2}$, compared with the normal fluorescence excitation spectrum, in the SE excitation spectra a sharp narrowing of lines is observed, the latter "exhibiting" thereby a greater selectivity of individual types of impurity centers in the matrix under study. These high-resolution SE spectra will allow more detailed studies of the spectral properties of certain types of impurity centers in a given matrix. Understanding the nature of the multiple components of SE may be useful for identifying the main types of impurity centers of pigments in cryogenic matrices. Moreover, such studies have a methodological significance and are of great importance as a method for selective low-temperature molecular spectroscopy.

\section{Hole-burning}

In this work we also carried out investigations of the photochemical properties of the $\mathrm{H}_{2} \mathrm{TBP} / \mathrm{N}_{2}$ system via a method involving the photoburning of stable spectral holes (persistent spectral hole burning-PSHB) in the $Q_{x}$ absorption band. The phenomenon of spectral hole-burning at low temperature is quite well known and has been studied in detail for the case of free base porphyrins (see Ref. 22 for a review). It is due to the intramolecular $\mathrm{NH}$-tautomerism, which in porphyrins implies only the displacement of the two central protons from one pair of opposite nitrogen atoms to the other (see structure in Fig. 1). ${ }^{23}$

The experimental results described previously have shown that the $Q_{x}(0-0)$ band of $\mathrm{H}_{2} \mathrm{TBP} / \mathrm{N}_{2}$ is structureless, i.e., inhomogeneously broadened due to the presence in the matrix of a large number of impurity centers and to the superposition of their different individual spectral bands. This inhomogeneous broadening can be eliminated through exposure to monochromatic laser light. Figure 5 presents the effect of such laser irradiation on the $Q_{x}$ band (Fig. 5(a)). The difference spectrum, obtained by subtracting the burnt spectrum from the unburnt one, is also shown (Fig. 5(b)). PSHB experiments then show us that burning at $\lambda_{\text {burn }}$ $=652.7 \mathrm{~nm}$ (Fig. 5(a)) forms a stable spectral hole corresponding to the zero-phonon line (zero-phonon hole), which can be saturated with increasing burning time. Typical laser exposure time for the observation of the spectral changes was chosen to be about $10 \mathrm{~min}$, and laser excitation power for the burning was $\sim 100 \mathrm{~mW}$. This burning time was sufficient to achieve saturation of the zero-phonon hole, which then resulted in its broadening up to $\sim 10 \mathrm{~cm}^{-1}$. As can be seen from Fig. 5, where the effect of laser excitation on the short-wavelength side of the ZPL is observed, there is a marked increase in absorption intensity due to the formation of a photoproduct (giving rise to an "antihole") on the highenergy side from $\lambda_{\text {burn }}$. The formation of an "antihole" during photoburning is associated with the formation of NH-tautomers of $\mathrm{H}_{2}$ TBP. This asymmetrical spectral distribution of the "antihole" due to the phototransformation of

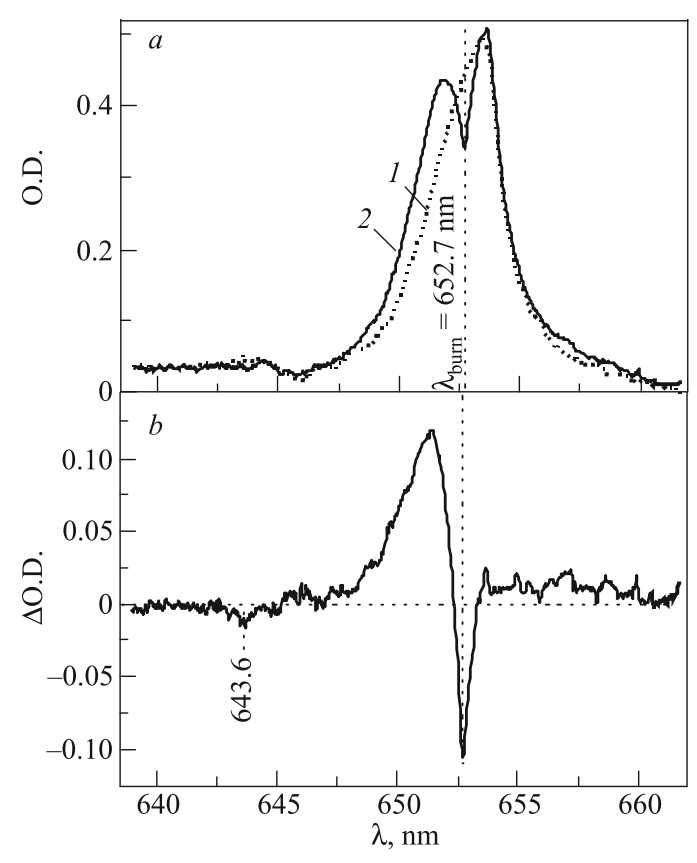

FIG. 5. (a) $Q_{x}(0-0)$ absorption band of $\mathrm{H}_{2}$ TBP molecules in a nitrogen matrix at $8 \mathrm{~K}$ before (1) and after burning (2), and (b) the difference of the two spectra showing a persistent spectral hole $\left(\lambda_{\text {burn }}=652.7 \mathrm{~nm}, P_{\text {burn }} \approx 100 \mathrm{~mW}\right.$, $t_{\text {burn }} \approx 10 \min$ ). 
$\mathrm{H}_{2}$ TBP symmetric molecules (point group symmetry $D_{2 h}$ ) is a consequence of the existence of a rather intense pseudophonon wing at long wavelengths resulting from the burning of impurity centers, which absorb laser radiation in the region of their phonon wing, i.e., centers with $\lambda_{Z P L}>\lambda_{\text {burn }}$. Naturally, at $\lambda_{\mathrm{exc}}=652.7 \mathrm{~nm}$ there would be more centers excited through their extensive phonon band than through their ZPL. As result, the long wavelength (with respect to the selected $\lambda_{\text {burn }}$ ) photoproduct absorption and the intense pseudo-phonon wing at $\lambda>\lambda_{\text {burn }}$ almost cancel out each other. It is essential to notice that similar spectral changes were also observed for the $Q_{x}(0-1)$ band located at $\sim 643.6 \mathrm{~nm}$, which means, for the band that corresponds to the electron-vibrational transition.

As it turned out in the case of $\mathrm{H}_{2} \mathrm{TBP} / \mathrm{N}_{2}$, the intersystem conversion in impurity centers has a rather low efficiency, which is the reason for the almost complete lack of influence of the photochemical transformation on the efficiency of the SE phenomenon.

\section{Conclusion}

The main results obtained in the present work on absorption, fluorescence (stimulated emission), and persistent spectral hole-burning of matrix-isolated molecules of $\mathrm{H}_{2}$ TBP in solid nitrogen can be summarized as follows: It was found that the exposure to pulsed laser excitation in the $Q_{x}$ and $Q_{y}$ absorption bands of $\mathrm{H}_{2}$ TBP molecules, trapped in the lowtemperature nitrogen matrix, induces the occurrence of stimulated emission, together with normal spontaneous emission of fluorescent $\mathrm{H}_{2}$ TBP molecules. This stimulated emission is evidenced by the strong enhancement of the intensity of one or more vibronic fluorescence lines, and it occurs when the intensity of the exciting laser radiation exceeds a certain threshold. The observation of such phenomenon means the achievement of a population inversion between $S_{1}(0-0)$ ground level and some vibronic sublevel of the ground electronic state. The vibronic transition involved in the process of stimulated emission corresponds to a vibrational level of $S_{0}$ state with an energy of $\sim 1615 \mathrm{~cm}^{-1}$, which is assigned to the stretching vibrations of $\mathrm{C}_{\alpha} \mathrm{C}_{m}$ methine bridges with $B_{1 g}$ symmetry.

The observation of stimulated emission was possible using pulsed laser excitation with nanosecond duration, not exceeding the lifetime of the lowest singlet $S_{1}$ state of $\mathrm{H}_{2} \mathrm{TBP}$, and a $10 \mathrm{~Hz}$ low repetition rate. In such a situation, the intersystem singlet-triplet conversion mechanism populating the lowest triplet state (a "trap" of the excited molecules) will only play a minor role, which is an important condition for the creation of population inversion in the system of singlet levels during the laser pulse.

The recorded spectra of the excitation of stimulated emission for individual impurity centers exhibit enhanced selectivity, which creates conditions for a more detailed study of the spectral properties of the molecules forming these impurity centers in the matrix.

The obtained results determine the scientific significance of these investigations. For instance, a practical significance of these results is our ability to identify opportunities for applications in the development of laboratory models of new thin-film materials based on low-temperature matrices colored with tetrapyrrole compounds for creating microlasers and light transformer devices (in the bluegreen region) from the monochromatic radiation in the red or the near infrared region. The results obtained can be used to expand the search for new microlaser media based on organic dyes.

This work was supported by BRFFR (projects F09F-001 and F11F-001) and CNRS France (Project Nos. 23181 and 24 692), and the Ulysses France-Ireland exchange (PHC program Ulysses No. 25709VA).

${ }^{a)}$ Email: jean-pierre.galaup@lac.u-psud.fr

${ }^{1}$ R. Reisfeld, E. Yariv, and H. Vinti, Opt. Mater. 8, 31 (1997).

${ }^{2}$ E. Yariv and R. Reisfeld, Opt. Mater. 13, 49 (1999).

${ }^{3}$ R. T. Kuznetsova, Yu. A. Manekina, E. N. Tel'minov, G. V. Mayer, S. M. Arabei, T. A. Pavich, and K. N. Solovyov, Opt. Atmos. Okeana 19, 653 (2006) [Atmos. Oceanis Opt. 19, 586 (2006)].

${ }^{4}$ R. T. Kuznetsova, G. V. Maier, Yu. A. Manekina, V. A. Svetlichnyi, E. N. Tel'minov, S. M. Arabei, T. A. Pavich, and K. N. Solovyov, Opt. Spektrosk. 102, 241 (2007) [Opt. Spectrosc. 102, 208 (2007)].

${ }^{5}$ R. T. Kuznetsova, G. V. Maier, Yu. A. Manekina, E. N. Tel'minov, S. M. Arabei, T. A. Pavich, and K. N. Solovyov, Kvantovaya Élektron. 37, 760 (2007) [Quantum Electron. 37, 760 (2007)].

${ }^{6}$ R. T. Kuznetsova, G. V. Mayer, Yu. A. Manekina, E. N. Tel'minov, S. M. Arabei, T. A. Pavich, and K. N. Solovyov, Opt. Spektrosk. 104, 223 (2008) [Opt. Spectrosc. 104, 190 (2008)].

${ }^{7}$ N. Dozova, C. Murray, J. G. McCaffrey, N. Shafizadeh, and C. Crépin, Phys. Chem. Chem. Phys. 10, 2167 (2008).

${ }^{8}$ C. Crépin, N. Shafizadeh, W. Chin, J.-P. Galaup, J. G. McCaffrey, and S. M. Arabei, Fiz. Nizk. Temp. 36, 563 (2010) [Low Temp. Phys. 36, 451 (2010)].

${ }^{9}$ P. P. Sorokin and J. R. Lankard, IBM J. Res. Dev. 10, 162 (1966).

${ }^{10}$ P. P. Sorokin, J. R. Lankard, E. C. Hammond, and V. L. Moruzzi, IBM J. Res. Dev. 11, 130 (1967).

${ }^{11}$ S. M. Arabei, K. N. Solovyov, and Yu. I. Tatulchenkov, Opt. Spektrosk. 73, 686 (1992) [Opt. Spectrosc. 73, 406 (1992)].

${ }^{12}$ I. E. Zaleski, V. N. Kotlov, A. N. Sevchenko, K. N. Solovyov, and S. F. Shkirman, Dokl. Akad. Nauk SSSR 207, 1314 (1972) [Proc. USSR Acad. Sci. Sov. Phys. Dokl. 17, 1183 (1973)].

${ }^{13}$ K. N. Solovyov, I. E. Zaleski, V. N. Kotlov, and S. F. Shkirman, Pisma Zh. Eksp. Teor. Fiz. 17, 463 (1973) [JETP Lett. 17, 332 (1973)].

${ }^{14}$ K. N. Solovyov, S. F. Shkirman, and T. F. Kachura, Izv. Akad. Nauk SSSR Ser. Fiz. 27, 767 (1963) [Bull. Acad. Sci. USSR, Phys. Ser. 27, 763 (1963)].

${ }^{15}$ A. N. Sevchenko, K. N. Solovyov, S. F. Shkirman, and T. F. Kachura, Dokl. Akad. Nauk SSSR 161, 1313 (1965) [Proc. USSR Acad. Sci. Sov. Phys. Dokl. 10, 349 (1965)].

${ }^{16}$ S. M. Arabei, Zh. Prikl. Spektrosk. 57, 56 (1992) [J. Appl. Spectrosc. 57, 572 (1992)].

${ }^{17}$ B. Ehrenberg and F. M. Johnson, Spectrochim. Acta, A 46, 1521 (1990).

${ }^{18}$ V. G. Maslov, Opt. Spektrosk. 50, 1091 (1981) [Opt. Spectrosc. 50, 599 (1981)].

${ }^{19}$ C. Murray, N. Dozova, J. G. McCaffrey, N. Shafizadeh, W. Chin, M. Broquier, and C. Crépin, Phys. Chem. Chem. Phys. 13, 17543 (2011).

${ }^{20}$ S. F. Shkirman, L. L. Gladkov, V. K. Konstantinova, and K. N. Solovyov, Zh. Prikl. Spektrosk. 66, 375 (1999) [J. Appl. Spectrosc. 66, 400 (1999)].

${ }^{21}$ V. A. Kuzmitsky, K. N. Solovyov, V. N. Knyukshto, I. K. Shushkevich, V. N. Kopranenkov, and A. M. Vorotnikov, Teor. Eksp. Khim. 19, 655 (1983) [Theor. Exp. Chem. 19, 604 (1984)].

${ }^{22}$ J.-P. Galaup, "Spectral selective studies of molecular doped solids and applications," in Advances in Multi-Photon Processes and Spectroscopy, edited by S. H. Lin, A. A. Villaeys, and Y. Fujimura (World Scientific, 2004), Vol. 16, p. 73.

${ }^{23}$ V. A. Kuzmitsky, A. N. Sevchenko, and K. N. Solovyov, Dokl. Akad. Nauk SSSR 239, 308 (1978) [Proc. USSR Acad. Sci. Sov. Phys. Dokl. 239, 194 (1978)].

This article was published in English in the original Russian journal. Reproduced here with stylistic changes by AIP. 